\title{
A Comparative Study of Angular Extrapolation in Sinogram and Stackgram Domains for Limited Angle Tomography
}

\author{
A.P. Happonen and U. Ruotsalainen \\ Institute of Signal Processing \\ Tampere University of Technology \\ P.O.Box 553, FI-33101 Tampere, Finland \\ \{antti.happonen, ulla.ruotsalainen\}@tut.fi
}

\begin{abstract}
In limited angle tomography, the projection views over a complete angular range of $180^{\circ}$ are not available for image reconstruction. The missing part of the projection or sinogram data need to be extrapolated numerically, if standard image reconstruction methods are applied. A novel stackgram domain can be regarded as an intermediate form of the sinogram and image domains, in which the signals along the sinusoidal trajectories of a sinogram can be processed independently. In this paper, we compare extrapolation of incomplete sinogram data in the sinogram and stackgram domains along the angular directions. The extrapolated signals are assumed to be band-limited, other a priori assumptions about the data are not made. In this study, we employed simulated numerical data with different ranges of the limited projection views. According to our experiments, extrapolation of the incomplete data in the stackgram domain provides quantitatively better results as compared to extrapolation in the sinogram domain. In addition, tangential degradation in the reconstructed images can not be observed in the case of stackgram extrapolation, in contrast to angular sinogram extrapolation.
\end{abstract}

\section{Introduction}

In tomography, measurement of a two-dimensional (2-D) cross-section of an object is represented as a sinogram with one-dimensional (1-D) projections. The sinogram is a $2-\mathrm{D}$ matrix representation, where the horizontal row refers to radial samples and the vertical column refers to evenly spaced angular views. A 2-D image of the projected cross-section is recovered from the sinogram data using image reconstruction. A reconstruction method such as filtered back-projection (FBP) 1] can be employed for this ill-posed inverse problem.

In this paper, we consider the problem of limited angle tomography, i.e. sinograms with incomplete ranges of projection views (Fig. 11). In practice, this kind of problem can arise, for example, in positron emission tomography (PET) imaging with BPET [2] or PENN-PET [3] tomograph. Without any restoration technique, the "naive" reconstruction (i.e. the missing projections are replaced with 
a

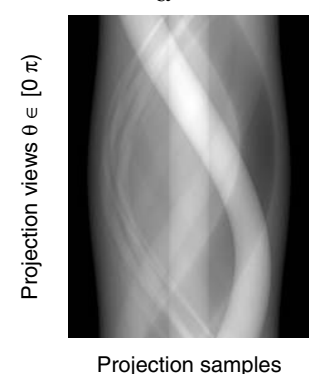

b

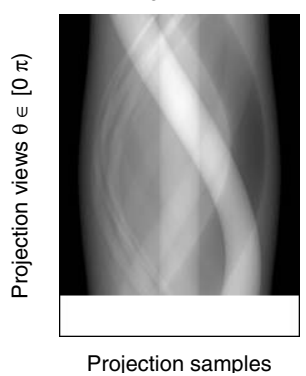

Fig. 1. Two sinograms illustrating limited angle problem in tomography: a) full range of projections; b) limited view of projections

zeros) of incomplete sinogram data does not provide quantitative images and introduces clear artifacts.

Some algorithms using a priori information about the sinogram data for extrapolation of the missing data have been introduced (e.g. 4 or [5]). Furthermore, comparisons of different algorithms for limited angle tomography problem have been published [6], 7]. Some of these algorithms do not extrapolate the missing projections of the sinogram directly in the sinogram domain, but incorporate a priori information e.g. in iterative image reconstruction [8]. In this paper, we compare extrapolation of the missing projections using an extrapolation technique based on the Gerchberg-Papoulis algorithm [9] in the sinogram and stackgram [10 11 domains along the angular directions. After extrapolation of the missing data, the sinograms can be reconstructed with common reconstruction algorithms.

Extrapolation of discrete signals can be seen as a signal filtering application. In the sinogram domain, filtering along the angular direction introduces tangential or non-uniform blurring to the reconstructed images [12. In contrast, the angular direction of the stackgrams can be exploited without introducing spatially varying blurring in the reconstructed images, according to our experimental investigation [13]. This suggests that extrapolation in the stackgram domain could be performed in a more appropriate way, and gives a motivation for this comparative study. In our experiments, we assumed that the signals to be extrapolated are band-limited. Additional a priori knowledge about the data was not exploited, in order to provide a fair comparison between the two domains.

\section{Background and Problem Formulation}

\subsection{The Stackgram Domain}

In the stackgram domain, the signals along the sinusoidal trajectory signals of the sinogram (see Fig. 2) can be processed without interaction with the crossing signals, in contrast to the sinogram domain. The mapping $S$ from the sinogram 

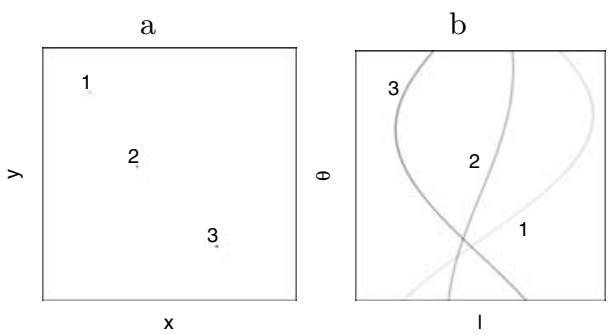

Fig. 2. An image and the corresponding sinogram: a) three points in the image domain $(x, y)$, and $\mathrm{b})$ their sinusoidal trajectories in the sinogram domain $(l, \theta)$

$g(l, \theta)$ into the stackgram domain $(x, y, \theta)$ can be defined in the continuous case as 10 .

$$
h(x, y, \theta)=S g(l, \theta)=g(x \cos \theta+y \sin \theta, \theta),
$$

where $\theta \in[0, \pi)$ and $(x, y) \in \mathbb{R}^{2}$. This mapping seems to be similar as the back-projection operator [1. In the equation (1), however, the back-projection integration from zero to $\pi$ along the $\theta$-direction is replaced by the third $\theta$ dimension resulting in the function $h(x, y, \theta)$, which forms the stackgram (Fig. 3).

In the stackgram, the values along the sinusoidal trajectory signals of the sinogram, or the locus-signals, are

$$
h_{\text {locus }}(\theta)=h(x, y, \theta) \text {, for each point }(x, y) \in \mathbb{R}^{2} \text {, }
$$

where $\theta \in[0, \pi)$.

An inverse of the stack operator $S_{\rho}^{-1}$ is a mapping from the stackgram domain $(x, y, \theta)$ into the sinogram domain $(l, \theta)$. This can be written with the weighted Radon transform as

$$
g(l, \theta)=S_{\rho}^{-1} h=\iint_{-\infty}^{\infty} \rho(x, y, l, \theta) h(x, y, \theta) \delta(x \cos \theta+y \sin \theta-l) d x d y
$$

where $l \in(-\infty, \infty), \theta \in[0, \pi)$, and $\rho$ is a weight function. The operator $S_{\rho}^{-1}$, denoted as the generalized inverse stack operator, maps each back-projected projection at the angle $\theta$ with the weight function $\rho$ into a $1-\mathrm{D}$ projection of the sinogram $g(l, \theta)$.

Discrete implementations of the both operators (Eq. 11 and 3) are described in the reference [13. The discrete stack operator is reversible, when it is implemented with the three-pass-rotation algorithm and sinc-interpolation [14].

\subsection{The Extrapolation Procedure}

Consider a discrete sinogram matrix $\mathbf{g}(l, \theta), l=0, \ldots, M-1$ and $\theta=0, \ldots, N-1$, where $l$ denotes the projection samples and $\theta$ the number of equally spaced projection views between $[0 \pi)$ radians. Similarly, let an array $\mathbf{h}(x, y, \theta), x, y=$ $0, \ldots, M-1$ and $\theta=0, \ldots, N-1$, be the corresponding discrete stackgram 


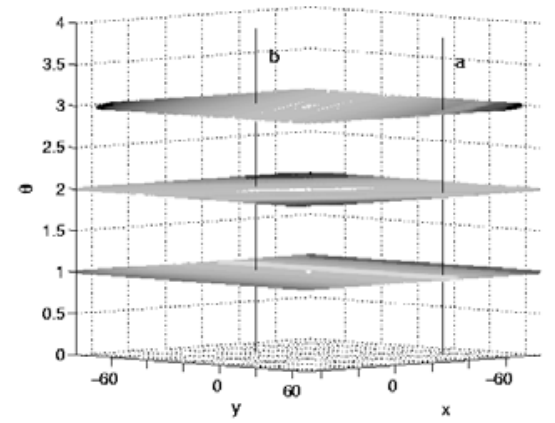

Fig. 3. An illustration of the stackgram $(x, y, \theta)$. The shown layers are back-projected from three projections of the sinogram. In the stackgram, signals parallel to the angular $\theta$-axis correspond to the signals along the sinusoidal trajectories of the sinogram (see Fig. 2). The lines $\mathbf{a}$ and $\mathbf{b}$ depict the locations of two different trajectory signals in the stackgram

(see Eq. 1). In limited angle tomography, the sinogram $\mathbf{g}(l, \theta)$, and therefore the stackgram $\mathbf{h}(x, y, \theta)$, are available only for the limited views $\theta=0, \ldots, L-$ 1 , where $L<N$. In this study, our objective is to extrapolate the missing projections $L, \ldots, N-1$ of an incomplete sinogram to a sinogram with a full range of projections $0, \ldots, N-1$ using the sinogram and stackgram domains.

We employ an extrapolation technique based on the well-known GerchbergPapoulis algorithm [9]. The applied extrapolation technique, however, is not recursive, and reduced to single matrix multiplication with the extrapolation matrix [15. The $N \times N$ extrapolation matrix $E$ can be constructed using an ideal low-pass filter matrix $B_{f c}$ with a cut-off frequency $f_{c}$ expressed in number of sample points in the discrete frequency domain. In addition, ones in a switching matrix $X=\operatorname{diag}\{0,0, \ldots, 0,1, \ldots, 1,1\}$ represent the missing part of the data $L, \ldots, N-1$. The extrapolation matrix E can be written as [15]

$$
E_{n}=\left(I-\left(X B_{f_{c}}\right)^{n+1}\right)\left(I-X B_{f_{c}}\right)^{-1},
$$

where $I$ is the identity matrix.

\section{Methods}

We compared extrapolation in the sinogram and stackgram domains along the angular $\theta$-directions. The stackgrams $\mathbf{h}(x, y, \theta)$ were transformed from the sinograms $\mathbf{g}(l, \theta)$ using the discrete stack-operator [13. Prior to the inverse transformation, the locus-signals of the stackgrams (Eq. 2) were extrapolated along the $\theta$-direction for each $(x, y)$ position. For comparison, the angular signals of the sinograms were extrapolated along the $\theta$-direction for each index $l$.

In this study, numerical sinogram data with four different ranges of missing projections (Fig. 4(a-d)) were generated for the comparison. The size of the sinograms were 192 in the radial samples, and 257 in the angular views. The 
a

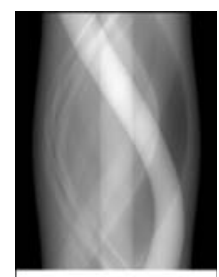

$\mathrm{b}$

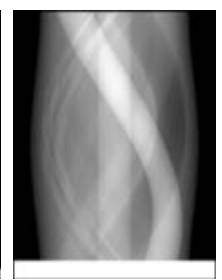

c

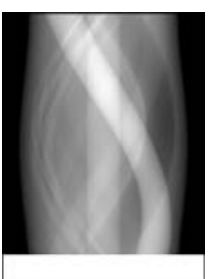

d

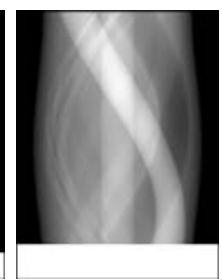

e

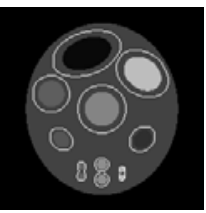

Fig. 4. The employed sinogram data in the study: a) 9 ; b) 17 ; c) 25 ; and d) 33 missing projections. In e), the shown ROI:s were used to evaluate with the MSE the performance of the extrapolation methods. For the error evaluation, all of the different ROI:s were regarded together as one ROI

numbers of the missing projections $L, \ldots, N-1$ were $9,17,25$, and 33 . The corresponding ranges of the projection views were $174^{\circ}, 168^{\circ}, 163^{\circ}$, and $157^{\circ}$, respectively, when the full range is 180 degrees (as e.g. in PET).

We applied the extrapolation matrices $E$ with 30 different cut-off frequencies $f_{c}$ (Eq. 4). The cut-off frequencies of the ideal low-pass filters were equally spaced from 0.004 to 0.117 (Nyqvist frequency 0.5). Extrapolation using these filters results in signals composed of sinusoidal curves of 1-30 different frequencies, since the extrapolation method (Eq. 4) can be seen as a sinusoidal fitting of discrete signals. It can be noticed that the condition of the extrapolation matrix $E$ is getting worse when the cut-off frequency (or the number of sample points in the discrete frequency domain) increases, because this introduces a more sparse diagonal for the matrix $E$. Similar effect happens if the signal to be extrapolated contains too many missing values. In our study, the matrix power $n$ was 500 (see Eq. 4), which corresponds to the number of iterations in the Gerchberg-Papoulis algorithm [9].

Evaluated images were reconstructed with the FBP-algorithm from the extrapolated sinograms. The reconstructed images were then evaluated with the mean square error (MSE) using a region of interest (ROI) shown in Fig. 4(e). Since extrapolation (as well as filtering) has different meaning in the angular directions of the sinogram and stackgram domains, 30 different extrapolation matrices, as described above, were applied. The cut-off frequencies versus the MSE values, or trade- off curves, are shown for the two extrapolation methods using the four incomplete sinograms. According to the evaluated curves, the resulting best sinograms and $\mathrm{FBP}$-images are shown as well.

\section{Results}

The evaluated trade- off curves with the four sinograms (Fig. 4(a-d)) for the compared methods are shown in Fig. 5. In Fig. 5, the constant curves in dashed line express the initial error levels, which were evaluated in such a manner that the missing projections were simply replaced by the zeros prior to image reconstruc- 
a

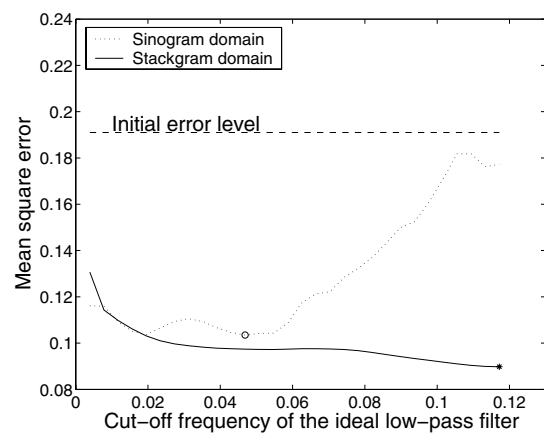

$\mathrm{c}$

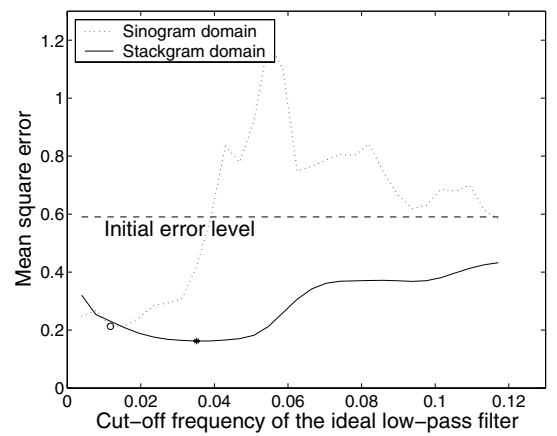

$\mathrm{b}$

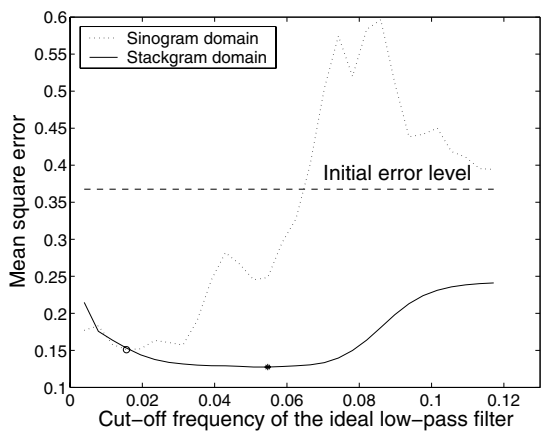

d

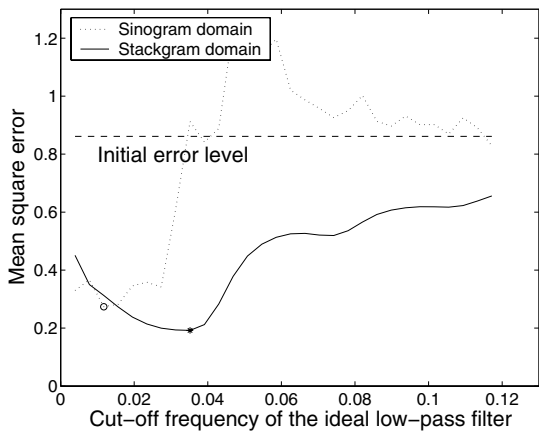

Fig. 5. The evaluated MSE:s of the reconstructed FBP-images versus the cut-off frequencies (Nyqvist frequency 0.5) of the applied ideal low-pass filters in extrapolation: a) 9 ; b) 17 ; c) 25 ; and d) 33 extrapolated projections. The constant dashed lines show the initial error for the images reconstructed from the incomplete sinogram data (see Fig. 4). The evaluated minimum values are shown with the circles and stars in the sinogram and stackgram cases, respectively. As regards to these curves, extrapolation in the stackgram domain provides better results. The corresponding best sinograms and reconstructed images are shown in Fig. 6 and 7

tion. As it can be noticed, stackgram extrapolation provides the best MSE values and smoother or more predictable trade-off curves for the two methods (Fig. [5), regardless of the range of missing projections. Besides, in the MSE sense, extrapolation in the stackgram domain seems to enable higher cut-off frequencies, and thus, resulting in more complex signals compared to sinogram extrapolation. As regards to the marked minimum values in Fig. 5, the corresponding best sinograms and FBP-images are shown in Fig. 6] and 7.

The sinogram of the full projection range is shown in Fig. 6)(a). Fig. 6)(b and d) shows the best extrapolated sinograms for the methods, according the minimum values of the curves in Fig. 5. The corresponding error sinogram images are shown in Fig. 6 (c and e), respectively. The shown sinograms are congruent with the trade-off curves (Fig. 5). Sinogram extrapolation (Fig. 66)(b)) introduces clearly less details (or frequencies) in the extrapolated projections, compared to stackgram extrapolation (Fig. 6(d)). 
a

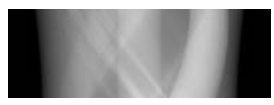

$\mathrm{b}$
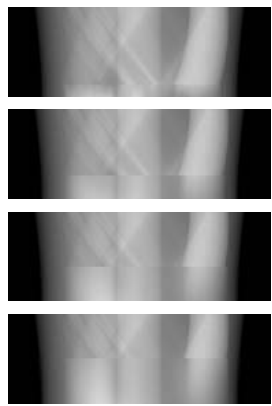

$\mathrm{c}$
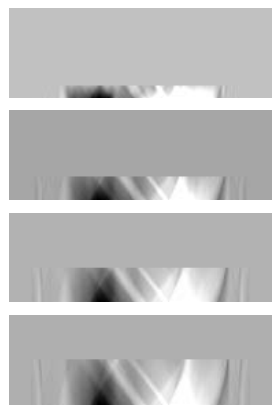

d
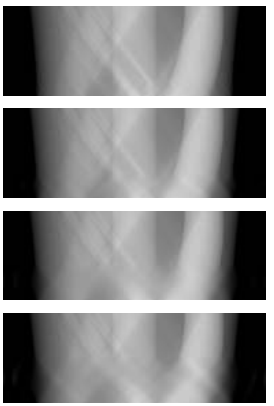

e
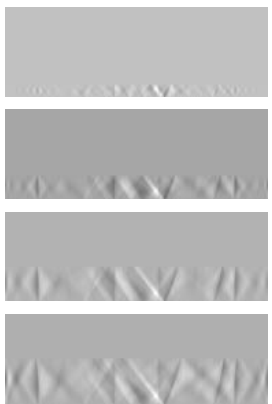

Fig. 6. Lower part of the sinograms: a) full range of projections; b) sinogram domain extrapolation; c) error images of b compared to a; d) stackgram domain extrapolation; e) error images of $d$ compared to $a$. In $b$ to e, the number of extrapolated projections were $9,17,25$, and 33 from top to bottom, respectively. The figure shows the best sinograms for the both methods, according to the Fig. 5 (see its marked minimum values). The reconstructed images are shown in Fig. 7 In stackgram domain extrapolation (d), the sinusoidal structure of the sinograms is preserved better, compared to sinogram domain extrapolation (b)

The reconstructed images from the sinograms (Fig. $6(a, b$, and d)) are shown in Fig. 7. Fig. 7(a) shows the image of the complete sinogram, while Fig. Z7(b) shows the images reconstructed with the "naive" FBP-algorithm from the incomplete sinogram data (Fig. $4(\mathrm{a}-\mathrm{d})$ ). At first, the resulting FBP-images of sinogram and stackgram extrapolation look similar (Fig. 17(c-d)). However, it can be noticed that the stackgram extrapolation introduces the lowest variations in gray level values, in comparison with both "naive" reconstruction and sinogram extrapolation (Fig. 7(b-c)). Besides, sinogram extrapolation causes tangential blurring in the reconstructed images, as expected, in contrast to stackgram extrapolation. This tangential distortion is more visible when the number of missing projections increases (Fig. Z7(c-d)).

\section{Discussion}

In this paper, we compare extrapolation of missing projection data for limited angle tomography in the sinogram and stackgram domains along their angular directions. Stackgram extrapolation performs quantitatively and visually better than sinogram extrapolation, according to the experiments. Extrapolation in the sinogram domain along the angular direction introduces observable tangential blurring in proportion to the incomplete range of missing projections (Fig. $7(\mathrm{c})$ ). Similar blurring effect is well-known in the case of angular sinogram filtering [12, therefore it is commonly avoided in noise reduction. According to 


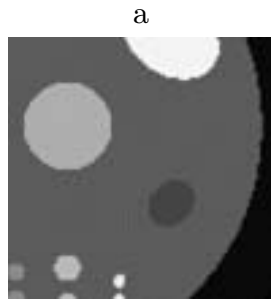

b) Limited view
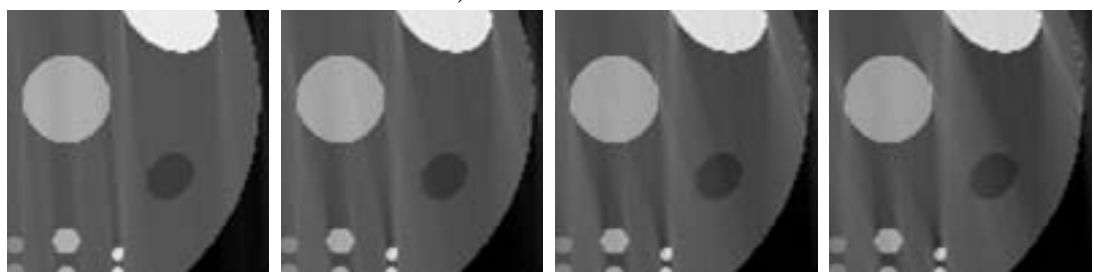

c) Sinogram extrapolation
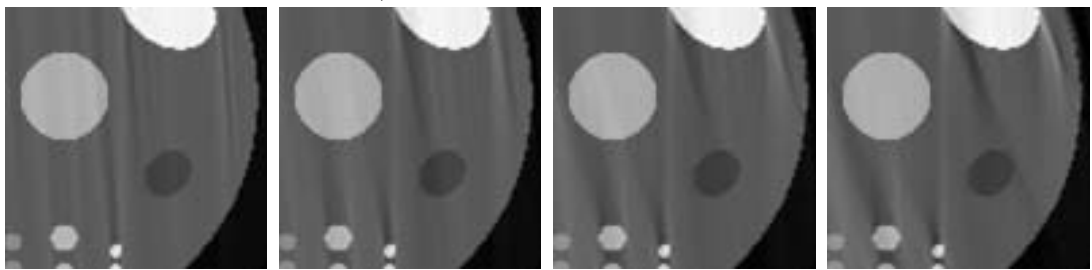

d) Stackgram extrapolation
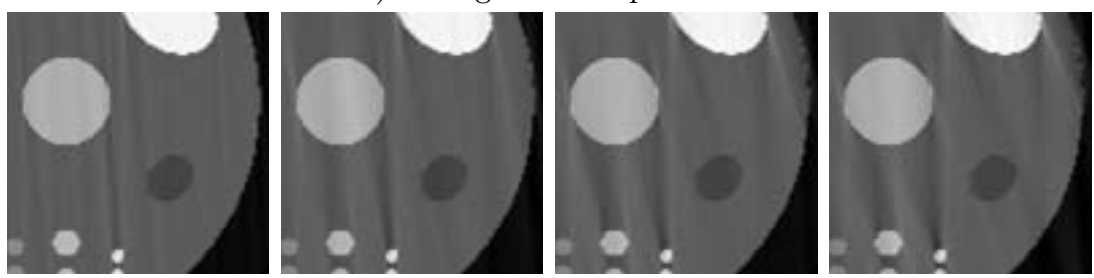

Fig. 7. A part of reconstructed FBP-images: a) full range of projections; b) limited projections; c) sinogram domain extrapolation; d) stackgram domain extrapolation. In b, the "naive" reconstruction from the incomplete sinogram data (see Fig. 4(a-d)), in which the number of missing projections were 9, 17, 25, and 33 from left to right, respectively. In c and d, the corresponding FBP-images from the extrapolated sinograms (see Fig. 6(b and d)). The best FBP-images are shown, according to the minimum values of the curves in Fig. 5. In c and d, the angular sinogram extrapolation introduces tangential distortions in the reconstructed images, unlike the angular stackgram extrapolation. It can be noticed that stackgram extrapolation provides quantitatively best results (the least variations in gray level values), as the curves (Fig. 5) also indicate. The images share a common gray scale

our experiments, the stackgram domain offers a more convenient extrapolation environment, since the tangential blurring cannot be observed in the reconstructed images (Fig. [7(d)). Our earlier filtering studies [13] support this finding as well. 
The sinogram $g(l, \theta)$ is symmetric, i.e. $g(l, \theta)=g(-l, \theta \pm \pi)$, and periodic in $\theta$ with period $2 \pi$, but not with $\pi[1$. Thus, in the discrete case $(l=0, \ldots, M-1$ and $\theta=0, \ldots, N-1)$, the values of the sinogram along the $\theta$-direction at 0 and $N-1$ can differ distinctly (one can verify this from Fig. 2). In the stackgram domain, on the other hand, the values of the locus-signals (Eq. 2) are periodic in $\theta$ with period $\pi$. The discrete Fourier transform, which is applied in the extrapolation algorithm (Eq. 4), considers the signals as periodic. This may explain the reason why the locus-signals, in contrast to the angular sinogram signals, can be extrapolated using the higher cut-off frequencies and resulting in better extrapolated signals (Fig. 55). However, the extrapolated parts of the signals contain low-frequencies or simple shapes, since the applied cut-off frequencies are relatively low (Fig. 5). The higher cut-off frequencies would introduce ill-posed matrix inverses (Eq. 4).

In this study, we did not use a priori knowledge about the data to be extrapolated. We employed noiseless numerical data in the experiments. In practice, e.g. in the case of PET data, the sinograms can be corrupted by the noise significantly. Therefore, more sophisticated extrapolation techniques for stackgram extrapolation need to be further studied.

\section{Conclusion}

We compared extrapolation of missing projections for limited angle tomography in the sinogram and stackgram domains along the angular directions. The extrapolated signals were assumed to be band-limited, additional a priori knowledge about the data were not exploited. Our experiments show, although further studies are still needed, that stackgram domain extrapolation can provide quantitatively and visually better results than sinogram domain extrapolation. In the stackgram extrapolation, the sinusoidal structure of the extrapolated projections can be restored better and the results are more predictable, compared to the sinogram extrapolation, according to our experiments. In limited angle tomography, the stackgram domain can offer a new potential approach for extrapolation of the missing projections from the incomplete sinogram data.

\section{Acknowledgments}

This work was supported by the Academy of Finland (project no. 104834).

\section{References}

1. Jain, A. In: Fundamentals of Digital Image Processing. Prentice-Hall International, Englewood Cliffs, NJ (1989) 434-448

2. Freifelder, R., Cardi, C., Grigoras, I., Saffer, J.R., Karp, J.S.: First results of a dedicated breast PET imager, BPET, using $\mathrm{NaI}(\mathrm{Tl})$ curve plate detectors. In: IEEE Nuclear Science Symposium Conference Record, 2001, San Diego, CA (2001) $1241-1245$ 
3. Karp, J., Muehllehner, G., Mankoff, D., Ordonez, C., Ollinger, J., DaubeWitherspoon, M., Haigh, A., Beerbohm, D.: Continuous-slice PENN-PET: a positron tomograph with volume imaging capability. Journal of Nuclear Medicine 31 (1990) 617-627

4. Yau, S.F., Wong, S.H.: A linear sinogram extrapolator for limited angle tomography. In: 3rd International Conference on Signal Processing, Beijing, China (1996) 386-389

5. Kazantsev, I.G., Matej, S., Lewitt, R.M.: Limited angle tomography and ridge functions. In: IEEE Nuclear Science Symposium Conference Record. (2002) 17061710

6. Ollinger, J.M., Karp, J.S.: An evaluation of three algorithms for reconstructing images from data with missing projections. IEEE Trans. Nucl. Sci. 35 (1988) 629-634

7. Oskoui, P., Stark, H.: A comparative study of three reconstruction methods for a limited-view computer tomography problem. IEEE Trans. Med. Imag. 8 (1989) 43-49

8. Delaney, A.H., Bresler, Y.: A fast and accurate fourier algorithm for iterative parallel-beam tomography. IEEE Trans. Image Processing 5 (1996) 740-753

9. Papoulis, A.: A new algorithm in spectral analysis and band-limited extrapolation. IEEE Trans. Circuits Syst. 22 (1975) 735-742

10. Happonen, A.P., Alenius, S.: Sinogram filtering using a stackgram domain. In: Proc. of the Second IASTED International Conference: Visualization, Imaging and Image Processing, Malaga, Spain (2002) 339-343

11. Happonen, A.P., Ruotsalainen, U.: Three-dimensional alignment of scans in a dynamic PET study using sinusoidal trajectory signals of a sinogram. IEEE Trans. Nucl. Sci. 51 (2004) 2620-2627

12. Daube-Witherspoon, M.E., Carson, R.E.: Investigation of angular smoothing of PET data. IEEE Trans. Nucl. Sci. 44 (1997) 2494-2499

13. Happonen, A.P., Alenius, S.: Investigation of sinogram filtering using stackgram domain. IEEE Trans. Med. Imag. (revised and awaiting the final decision) (2005)

14. Unser, M., Thevenaz, P., Yaroslavsky, L.: Convolution-based interpolation for fast, high quality rotation of images. IEEE Trans. Image Processing 4 (1995) 1371-1381

15. Sabri, M.S., Steenaart, W.: An approach to band-limited signal extrapolation: The extrapolation matrix. IEEE Trans. Circuits Syst. 25 (1978) 74-78 